\title{
Optimal Utilization of Rebar Stock for Cutting Processes in Housing Project
}

\author{
Asst. Prof. Dr. Sawsan Sabeeh Al-Zubaidy, Salam Qaddoori Dawood ${ }^{1}$, Israa Dhiaa Khalaf ${ }^{2}$ \\ Production Engineering and Metallurgy Department / Industrial Engineering, University of Technology, Baghdad, \\ Iraq $^{1,2}$
}

\begin{abstract}
The cutting processes of rebar stock in housing project cause a large amount of waste because of high variety between required rebar lengths and standard rebar lengths, this problem is called one dimensional cutting stock problem. This research aims to utilize the rebar stock and minimize the losses using integer linear programming approach- aided by Advanced Interactive Mathematical Modeling System (AIMMS) software for solving the problems. These one- dimensional cutting rebar stock problems are divided into 19- problem according to the variety in the rebar diameter $(25 \mathrm{~mm}, 16 \mathrm{~mm}, 12 \mathrm{~mm}$, and $10 \mathrm{~mm}$ ), and the variety in the design of activities in that housing project. The losses that are produced for each activity are utilized in the next activity when the length of item losses is larger than the required small item lengths in next activity for the same rebar diameters. That makes the utilization is to be maximized, where the Utilization Stock Ratio is reached to (98.53\%).
\end{abstract}

Keywords: One- Dimensional Cutting Stock Problem; Integer Linear Programming; and Optimal Solutions.

\section{INTRODUCTION}

Cutting Stock Problems (CSPs) are the combination of two problems; the first one is the assortment problem, and the second one is the trim loss problem. When small pieces are being cut out from large objects, these two problems arise. The assortment problem addresses the issue of choosing proper dimensions for the large objects. The Trim Loss problem addresses the issue of how to cut out the small items from the given large objects in such a way that wastage material will be minimized [1].

One- Dimensional Cutting Stock Problem (1D- CSP) one of the important problems, the problem in its simplest form can be described as follows. Given materials that are available in certain lengths, cut them in order to generate certain required lengths. For instance, assume that we are cutting rebar to desired lengths. Being a one-dimensional problem, all rebar are of the same diameter and only their respective lengths differ.

On the one hand, there are different lengths of available rods that we have in stock; while on the other hand; there are lengths of desired rebar that are demanded by customers. A cutting plan, consisting of cutting patterns, will determine how to generate the required rebar from the available rebar. Suppose that there are many available lengths for which there are number of supplies of these available rods. On the other hand, there are required lengths for which there are. The cutting plan is assumed to consist of a number of distinct cutting patterns that are enumerated from plan to another [2].

There are two kinds of stocks in (1D-CSP) [3]:

a. A Standard One-Dimensional Cutting Stock Problem (S1D-CSP) is known as an Non- Deterministic Polynomial (NP) complete one, as shown in Figure (1.a).
The assumptions of this problem are as follows:

- All used stock lengths should be cut to the end in as much as it is possible.

- All stock lengths are identical (with same lengths).

Application of this problem is available in pipes, steel trimming, and film rolls cutting.

b. A General One-Dimensional Stock Cutting Problem (G1D-CSP), as another kind of the one dimensional problem, trim losses can be used later. The storage stocks have different lengths. Reduction of cutting wastes is one of the main goals in the cutting process and also one of the basic purposes in the 1D-CSP [4]. As shown in Figure (1.b). Application of this problem is available in steel structure industries, wood strips cutting, and so on.

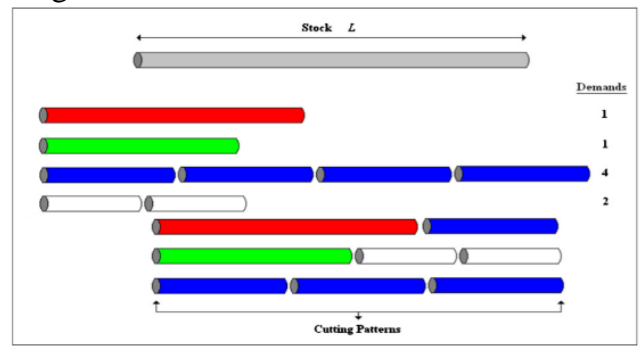

a- S1D-CSP

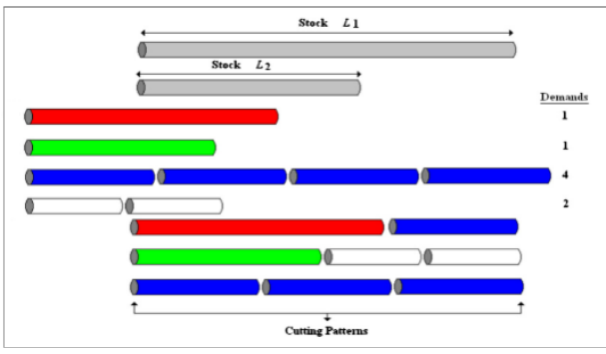

b- G1D-CSP

Figure 1: One-Dimensional Cutting Stock Problem (1D-

CSP) [5]. 


\section{LITRITURE REVIEW}

This section presents different studies published in the last years that utilize different methods that overcome the complexity of Cutting Stock Problems (CSPs):Shahinet et al. (2004) [6]: present a genetic algorithm (GA) model for solving the one-dimensional CSP (GA1D). In the United States, vast amounts of construction waste are produced every year. Construction waste accounts for a significant portion of the municipal waste stream of the United States. One-dimensional stocks are one of the major contributors to construction waste. The traditional optimization techniques (i.e., linear programming and integer programming) are used to solve the one-dimensional cutting stock problem (CSP).The comparison shows a high potential of savings that could be achieved.

Zuchao and Gang (2005) [7]: present a multi-stage genetic algorithm for approximately solving the one-dimensional cutting stock problem with only one stock length (for short, 1D-CSP). The objective is to maximize the utilization of the stock. The salient features of this algorithm are the optimizing way in which genetic algorithm performs unsaturated optimization at each stage. The efficiency of the proposed algorithm is demonstrated by several numerical results.

Gleb and Guntram (2007) [8]: designed a sequential heuristic to minimize material input and show its high effectiveness for this purpose. Then they extend it to restrict the number of open stacks to any given limit. Then, the heuristic is simplified and integrated into a setup minimization approach in order to combine setup and open-stacks minimization for 1D-CSP. To get a smaller number of open stacks, they split up the problem into several parts of smaller sizes. Different solutions are evaluated in relation to the multiple objectives using the Pareto criterion.

\section{METHOD FOR SOLVE TRIM LOSS MINIMIZATION PROBLEM}

It is possible to solve the trim loss minimization problem by linear programming without first enumerating every feasible slitting pattern. This is extremely important because a large number of feasible patterns may exist when narrow widths are to be slit from a wide stock roll. Pierce (1964) showed that in such situations the number of slitting patterns can easily run into the millions. Because only a small fraction of all possible cutting patterns need to be considered in finding the minimum trim loss solution, the delayed pattern generation technique is developed to solve trim loss minimization problems in much less time than would be required if all the slitting patterns were input to a general purpose linear programming algorithm [9].

The LP optimal solution, which generally is non-integer, has associated a small fraction of all possible patterns. To obtain an integer solution should solve a final integer CSP after using an extra column-generation procedure [10].In general; the CSP with all its extensions and variants has been classified as NP-hard. The CSP is essentially an integer programming problem; however, a two stage approach involving an LP relaxation of the CSP at the first stage followed by a rounding-up procedure at the second stage can be applied for many variants of the CSP. This approach is frequently used for solving CSPs by applying the column generation method of Gilmore and Gomory, and an appropriate rounding of the solution of the continuous relaxation problem. An auxiliary problem arises from the LP formulation where the columns of the LP constraint matrix need to be determined. The columns of the LP constraint matrix represent all the cut patterns (i.e. the different ways of cutting the material) that can be produced from the available stock material. Columns may be generated in two ways: in advance or on-line. Advance column generation is used when all of the feasible patterns are being generated for small to medium problems. It is also used only when a representative subset is being generated for a large problem. The on-line pattern generation is used for solving large integer problems by using a column generation technique similar to that of the classic one-dimensional CSP. For the one-dimensional CSP, Gilmore and Gomory used an impressive column generation technique built into the frame of the simplex method. One of the factors that add to the complexity of the CSP is the large number of cutting patterns that may be encountered. When the CSP is expressed as an integerprogramming problem, the large number of cutting patterns involved generally makes computation infeasible [11].

\section{PROBLEM DESCRIPTIONS}

Integer linear programming that is aided by Advanced Interactive Mathematical Modeling System (AIMMS) software approach for solving research problems has been applied in the actual process environment at Wassit province-Al Aziziyah city in housing project which is being to be achieved by Al-Mansour Company for Building and Construction. The cutting process of reinforcement steel (Rebar) was found to occurs randomly in the project location; engineers did not have clear plans or methods for the trimming processes that cause a large loss in the stocks; therefore a plan was made for saving the stocks. So we will present the environment overview, practical side, and apply ILP- based on software for solving One-Dimensional Cutting Stock Problem (1DCSP).

Solution of Rebar- Cutting Stock Problem divided according to four diameter category problems which were used $(25 \mathrm{~mm}, 16 \mathrm{~mm}, 12 \mathrm{~mm}$, and $10 \mathrm{~mm})$ - category problem. Each problem can be subdivided and solved according to basic building activity designs (foundation, columns, beams, slabs, stairs, balcony, and front arch building), when the problem is solved and trim losses are minimized in a current activity design, it should check the trim loss lengths in that activity designs, where the trim 
loss lengths are shorter than these required item lengths in next activity designs, it will be considered a waste, if these trim loss lengths equal or longer required item lengths in next activity designs, it should be stored and utilized next activity designs to get optimal utilization stock ratios.

Residential complexes $\mathrm{n}$ that projecti are divided into two different types according to the design and size. Building type (A) consists of (23) units with (207) apartments, while building type (B) consists of (41) units with (246) apartments. Each unit has two apartments of 150 square meters in each of the three floors. so building type (B) will be taken as a case study because building type (B) unit numbers are larger than that of building type (A). Experience factor differs from one engineer to another so It needs to determine the rebar amounts, also minimize trim losses, Al- Mansour company reaches to Utilization Stock Ratios (USR) equals approximately (93\%).

\section{IMPLEMENTATION OFINTEGER LINEAR PROGRAMMING ON HOUSING PROJECT}

We determined all quantities of required items with different lengths and diameters that are used in Onebuilding construction for all activities of which are presented mainly four of them in the figure 2, and we have grouped their items which will cutby two workers within different durations using mechanical cutter.

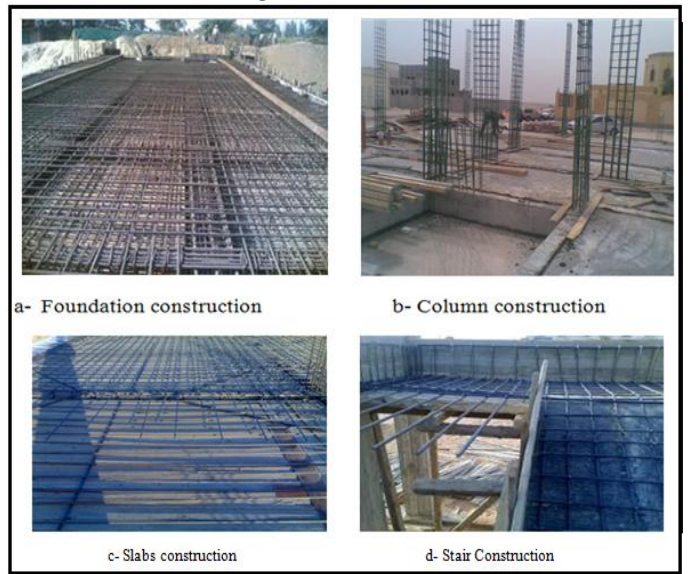

Figure 2: Different construction activities of building

These required quantities of rebar are distributed into different structural designs with variety amounts for each activity design; it will be illustrated as shown in the table I.

TABLE I: Illustrates The Activities That are Needed Rebar.

\begin{tabular}{|c|c|c|c|c|}
\hline \multicolumn{4}{|c|}{ Diameter Category } & \multirow[t]{2}{*}{ Activity } \\
\hline $25 \mathrm{~mm}$ & $16 \mathrm{~mm}$ & $12 \mathrm{~mm}$ & $10 \mathrm{~mm}$ & \\
\hline Required & Required & & & Foundation \\
\hline Required & Required & & Required & Columns \\
\hline & Required & Required & Required & Beams \\
\hline & Required & Required & Required & Slabs \\
\hline & Required & Required & Required & Stairs \\
\hline & & Required & Required & Balcony \\
\hline & Required & Required & Required & Front- Arch \\
\hline
\end{tabular}

A.

Problem Limitations:

Generally, these problems have identical large objects (I stocks) with one dimension (length (L)), and the required items will be assigned to the large objects which will fulfill the required orders $(\mathbf{V})$, so the varieties will be in the nature of item quantities and lengths (M, F, R, C) according to Dychoff's typology to determine the stock should be used and minimize the trim losses, we should identify the inputs and variables of the problem in symbols to simplify the explanation of typology as depicted below:

1) Large objects (stocks) lengths (L) are constant.

2) The quantities of required small items $(\mathrm{Rk}=\mathrm{R} 1, \ldots \mathrm{Rs})$.

3) Identical length for each quantity $(\mathrm{lk}=11, \ldots \mathrm{ls})$.

4) Ordered small items $(i=1, \ldots n)$.

5) Small item lengths $(\mathrm{li}=11, \ldots \ln )$.

6) The item lengths number in such stock number (lij).

7) Cutting Patterns $(c=1, \ldots p)$.

8) Total trim Loss $\left(\sum \mathrm{T}\right)$

9) Average length $(\mathrm{Al})$.

10) The total number of stocks to be cut $(X)$ ranging according to patterns $(\mathrm{Xc}=\mathrm{X} 1, \mathrm{X} 2 \ldots \mathrm{Xp})$, where $\mathrm{m}=\sum_{\mathrm{c}=1}^{\mathrm{p}} \mathrm{Xc}$, and $\mathrm{z}=\mathrm{min} \sum_{\mathrm{c}=1}^{\mathrm{p}} \mathrm{Xc}$.

Figure 1.3 below represents the problem limitations:

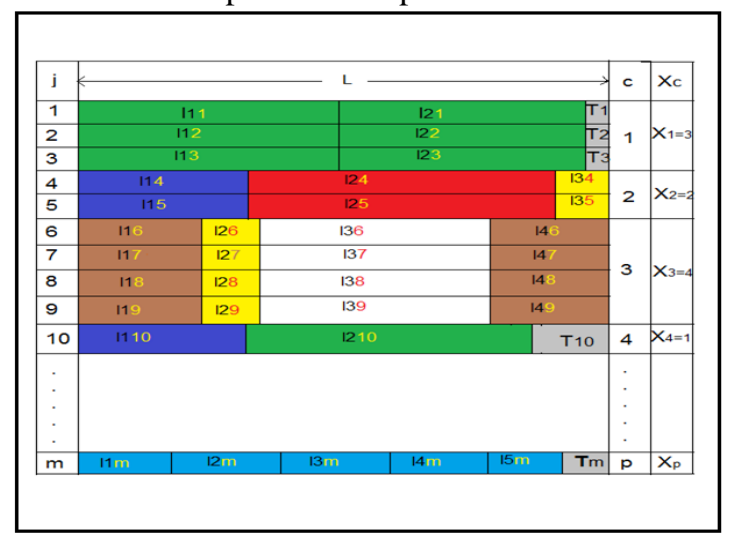

Figure 3:The problem limitations.

These different colors on the previous shape represent different number of item quantities ranging from $\mathrm{Rk}$ to $\mathrm{Rs}$ (R1=7, R2=3， R3=2， R4=6， R5=8， R6=4, $\mathrm{R} 7=5, \ldots \mathrm{Rs})$. While the gray color represents trim losses in different numbers of used stocks with different loss lengths. Utilization Stock Ratio (USR) $=\frac{\sum_{\mathrm{i}=1}^{\mathrm{n}} \mathrm{li}}{\mathrm{m} * \mathrm{~L}} \times 100 \%$ 1)

B. Data Insertion in AIMMS Software and Problem Solutions: 
When the system has been opened, the scheduler need to insert data of the problems in the data table which consists of rows and columns, there are two basic rows which represent the required item lengths (finals) and the demand quantities in addition to standard stock length. This screen shows existing data which were entered to simplify the data insertion to the beginners, and the user should delete these data, and then insert our problem data by pressing insert key button for each new item should be added.

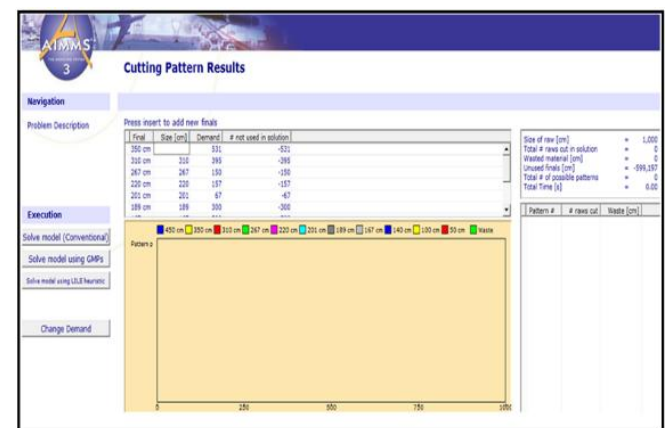

Figure 4: Data Insertion in the software system.

The user can insert a new value instead of the previous value which belongs to stock length (standard length). This appears on the right side of the screen shoot above. After insertion of the cutting stock lengths, the number of demands, the required item lengths, and quantities value, the software will be ready to solve the problem. The problem is solved by one click on the button (solve model) which appears on left side of the previous screen shoot [12].

The problems solution can be divided according to diameter categories ( $25 \mathrm{~mm}, 16 \mathrm{~mm}, 12 \mathrm{~mm}$, and $10 \mathrm{~mm}$ ). In addition to this problem solution classification, the building activities are considered as sub- division to solve the problems; these problems have different typologies, different required numbers, different total required lengths, and different item numbers.

After finishing solution of each problem, trim losses occur and these trim losses differ from problem and another, some of these trim losses can used to fulfill next required items in certain activity design with same diameter category after storing these trim losses, to save the stock and utilize as mentioned previously Utilization Stock Ratio, so tableII will illustrate all 19 problem solution results and table III will summarize the trim losses which can store to utilize as shown below:

TABLE II: Problem Solution Results

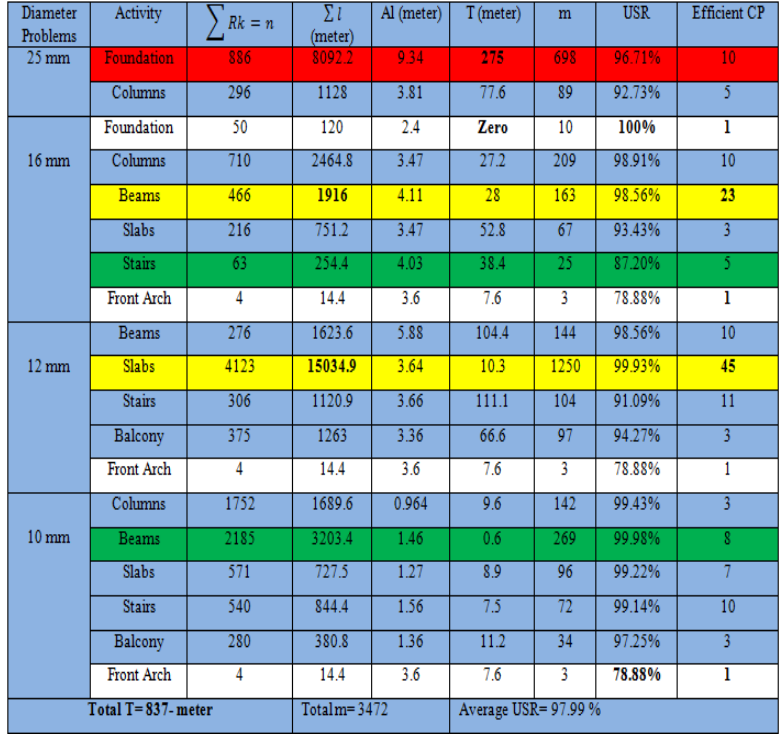

TABLE III: Utilization of Trim Losses to Fulfill Next Required Items of Activity Designs

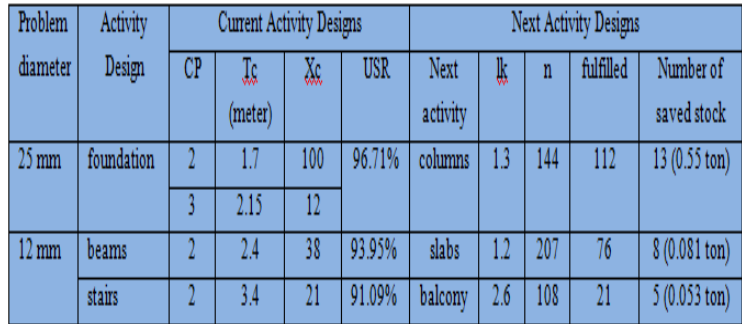

The trim losses are utilized in $(25 \mathrm{~mm}$ and $12 \mathrm{~mm}$ ) diameter problems, and the total trim losses reduced to (610- meter) for all problems instead of (837- meter), the utilization stock ratio increased to $(\mathbf{9 8 . 5 3 \%})$ instead of $(\mathbf{9 8 . 1 4 \%})$. there is no utilization for trim losses that occur in $(16 \mathrm{~mm}$ and $10 \mathrm{~mm})$ diameter problems because of having lengths shorter the next required item lengths.

\section{DISCUSSION AND EVALUATION}

The evaluation of the problem results could be summarized as the following:

- Total trim losses for each problem can be minimized by storage it to be utilized in the next activity, that can be applied in different problems. So the total trim losses for all problems are minimized to (610 meter) by the utilizing in other activities and the total number of used stock reduced with utilization stock ratio $(\mathbf{9 8 . 5 3 \% )}$.

- Largest trim losses was 275 meters in the foundation $(25 \mathrm{~mm})$ problem $(1 \backslash \mathrm{V} \backslash \mathrm{I} \backslash \mathrm{R})$, that lose due to the variety among the lengths and required items in addition to the average lengths (AI) is larger than the remaining average lengths of other problems.

- Largest and smallest utilization stock ratios (USR) were in the foundation $(16 \mathrm{~mm})$ problem $(100 \%)$ and front arch (16 mm, 12mm, and $10 \mathrm{~mm})$ problem $(78.88 \%)$. That means the congruent required item lengths do not give an indicator that refers to minimize trim losses, while the suitable stock length which is divided into congruent required item lengths 
considered effective factor to minimize the trim losses and utilize the stock in addition to there is no choices to generate more of effective cutting patterns (only 1 cutting pattern).

- Largest number of used stocks was in the slabs (12 $\mathrm{mm})$ problem $(\mathrm{m}=1250)$ because of needing for much of required items $(n=15034.9)$ with many different required item lengths $(\mathrm{k}=24)$, so that needs to generate more of efficient cutting patterns (45), it is considered $(1 \backslash \mathrm{V} \backslash \mathrm{I} \backslash \mathrm{M})$, also beams $(16 \mathrm{~mm})$ problem is $(1 \backslash \mathrm{V} \backslash \mathrm{I} \backslash \mathbf{F})$ problem, so it is need to generate more of efficient cutting patterns (23).

- There are varieties among solution results in the same problem typology such as $(1 \backslash \mathrm{V} \backslash \mathrm{I} \backslash \mathrm{R})$ as noted in the stairs $(16 \mathrm{~mm})$ problem and beams $(10 \mathrm{~mm})$ problem solution results, where the utilization stock ratio in the stairs $(16 \mathrm{~mm})$ problem result was $(\mathrm{USR}=87.20 \%)$, while utilization stock ratio in the beams $(10 \mathrm{~mm})$ problem result was (USR $=99.98 \%)$.

\section{CONCLUSIONS AND FURTHER RESEARCH}

The cutting patterns that are generated to fulfill the required items are increased, when there is high variety among the small item lengths, quantities and the large object length.

1. Trim loss depends on the nature of small item lengths, where the trim loss will be decreased whenever these small item lengths are having common factors or halves of common factors for the large object lengths, this helps to get optimal solutions as well as generate fast cutting patterns.

2. Utilization Stock Ratios (USR) reach to $(98.53 \%)$ by using this approach, while the (USR) that company reaches lower than this percentage approximately $(93 \%)$

3. All these variables $\left(\mathrm{n}, \mathrm{k}, \sum_{\mathrm{i}}^{\mathrm{n}} \mathrm{li}, \mathrm{Al}\right)$ do not give an indicator for increment or decrement in trim losses (T). On the other hand, the used stock (large objects) will be increased whenever these variables are increased.

4. This approach (ILP- aided by AIMMS software) can be used by the company or the ministry for all building projects and construction which depend on reinforcement steel to get future outlooks.

5. It is important to store trim loss amounts to utilize it in other activities that have lengths less than or equal to these trim loss lengths.

6. Generally, whenever the required item lengths were small, the total trim lose lengths will be small.

In the future studies, we recommend the following:

1. Study of the variety of cutting pattern generation effects on setup times and labor costs.

2. Study the effect of diversity between required item

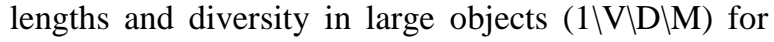
patterns generation.

\section{REFERENCES}

[1]. S. Eroglu, and B. Noche, "Slitting Optimization Problem", Sim.Serv, 1995 .

[2]. H. Eiselt, and C. Sandblom, "Linear Programming and its Applications", Springer-Verlag Berlin Heidelberg, 2007.

[3]. H. Terashima-Mar'in, P. Ross, and M. Valenzuela-Rend'on, "A GA-Based Method to Produce Generalized Hyper-heuristics for the 2D-Regular Cutting Stock Problem", GECCO'06, Seattle, Washington, USA, , July 8-12, 2006.

[4]. M. Jahromi, R. Tavakkoli-Moghaddam, A. Makui, and A. Shamsi, "Solving an One-Dimensional Cutting Stock Problem by Simulated Annealing and Tabu Search", Journal of Industrial Engineering International, licensee Springer Open, 2012.

[5]. M. Berberler, Nuriyev, and A. Yildirım, "A Software for the OneDimensional Cutting Stock Problem", Journal of King Saud University (Science), VOL. 23, pp. 69-76, 2011.

[6]. Y. Khalifa, O. Salem, and A. Shahin, "Cutting Stock Waste Reduction Using Genetic Algorithms", GECCO'06, Seattle, Washington, USA, 2004.

[7]. Z.Wang, and G. Rao, "A Multi-stage Genetic Algorithm for Onedimensional Cutting Stock Problems with One Stock Material Length", Journal of Systems Science \& Information, Vol. 3 Issue 3, Sep., 2005.

[8]. G. Belov, and G. Scheithauer, "Setup and Open-Stacks Minimization in One-Dimensional Stock Cutting", INFORMS Journal on Computing, Vol. 19, No. 1, pp. 27-35, 2007.

[9]. R. Haessler, and P. Sweeney, "Cutting Stock Problems and Solution Procedures", European Journal of Operational Research, VOL. 54, pp. 141-150, 1991.

[10]. L. Fernandez, L. A. Fernandez, and C. Pola, "Integer Solutions to Cutting Stock Problems", 2nd International Conference on Engineering Optimization, Lisbon, Portugal, September 6-9, 2010.

[11]. S. Suliman, "Pattern Generating Procedure for the Cutting Stock Problem", Int. J. Production Economics VOL 74, pp. 293-301, 2001.

[12]. Available at: http://www.aimms.com 